\title{
Evaluación del desempeño pronóstico de dos puntajes de predicción de mortalidad a siete días en pacientes adultos oncológicos críticamente enfermos admitidos en una unidad de cuidados intensivos
}

\author{
Prognostic performance assessment of two \\ seven-day mortality prediction scores in oncologic \\ critically ill adult patients admitted to an intensive \\ care unit
}

\author{
Ingrid Tatiana Rojas, Arnold Méndez, Fernando José Rincón \\ - Bogotá. D.C. (Colombia)
}

\section{Resumen}

Introducción: los sistemas de puntuación para predicción se han desarrollado para medir la severidad de la enfermedad y el pronóstico de los pacientes en la unidad de cuidados intensivos. Son útiles para la toma de decisiones clínicas, la estandarización de la investigación y la comparación de la calidad de la atención.

Material y métodos: estudio observacional analítico de cohorte en el que revisaron las historias clínicas de 283 pacientes oncológicos admitidos en la unidad de cuidados intensivos (UCI) del Centro de Investigaciones Oncológicas CIOSAD, durante enero de 2014 a enero de 2016, a quienes se les estimó la probabilidad de mortalidad con los puntajes pronósticos APACHE IV y MPM II, se realizó regresión logística binaria con las variables de los modelos en sus estudios originales, se determinó calibración, discriminación y se calcularon criterios de información Akaike AIC y Bayesiano BIC.

Resultados: en la evaluación de desempeño de los puntajes pronósticos APACHE IV mostró mayor capacidad de predicción $(\mathrm{AUC}=0.95)$ versus MPM II $(\mathrm{AUC}=0.78)$, los dos modelos mostraron adecuada calibración con estadístico de Hosmer y Lemeshow para APACHE IV $(p=0.39)$ y para MPM II ( $p=0.99)$. El delta BIC es 2.9 mostrando evidencia positiva en contra de APACHE IV. El estadístico AIC es menor para APACHE IV indicando que es el puntaje con mejor ajuste a los datos.

Conclusiones: APACHE IV tiene un buen desempeño en la predicción de mortalidad de pacientes oncológicos críticamente enfermos. Es una herramienta útil para el clínico en su labor diaria. al permitirle distinguir los pacientes con alta probabilidad de mortalidad. (Acta Med Colomb 2018; 43: 81-89).

Palabras clave: puntaje pronóstico, cuidado crítico, cáncer, mortalidad.

\footnotetext{
Abstract

Introduction: scoring systems for prediction have been developed to measure the severity of the disease and the prognosis of patients in the intensive care unit. They are useful for clinical decision-making, standardizing research and comparing the quality of care.

Materials and Methods: an observational cohort analytical study in which the medical records of 283 oncological patients admitted to the intensive care unit (ICU) of the CIOSAD Oncology Research Center from January 2014 to January 2016 were reviewed. The probability of mortality with the APACHE IV and MPM II prognostic scores was estimated, binary logistic regression was performed with the variables of the models in their original studies, calibration, discrimination was determined and Akaike AIC and Bayesian BIC information criteria were calculated.
}

Ingrid Tatiana Rojas Ruiz: Enfermera, Especialista Calidad en Salud, Especialista y Magister en Epidemiología. Profesor Auxiliar Universidad Nacional de Colombia Sede Bogotá, Facultad de Enfermería, Departamento de Enfermería; Dr. Arnold Méndez Toro: Médico y Cirujano, Universidad Nacional de Colombia. Especialista en Medicina Interna, Universidad Nacional de Colombia. Especialista Cardiología, Universidad Militar Nueva Granada; Dr. Fernando José Rincón: Médico y Cirujano, Universidad Nueva Granada. Especialista Medicina Interna, Pontificia Universidad Javeriana. Coordinador UCI IPS Centro de Investigaciones Oncológicas, Clínica San Diego. Bogotá. D.C. (Colombia). Correspondencia: Dra. Ingrid Tatiana Rojas Ruiz. Bogotá. D.C. (Colombia). E-mail: itrojasr@unal.edu.co Recibido: 27/IX/2016 Aceptado: 03/IV/2018 
Results: in the evaluation of the performance of the prediction scores,APACHE IV showed greater predictive capacity (AUC $=0.95$ ) versus MPM II (AUC $=0.78)$; the two models showed adequate calibration with Hosmer and Lemeshow statistics for APACHE IV ( $p=0.39)$ and for MPM II ( $p$ = 0.99). The BIC delta is 2.9 showing positive evidence against APACHE IV. The AIC statistic is lower for APACHE IV indicating that it is the score with the best fit to the data.

Conclusions: APACHE IV has a good performance in the prediction of mortality of critically ill oncologic patients. It is a useful tool for the clinician in his daily work, allowing him to distinguish patients with a high probability of mortality. (Acta Med Colomb 2018; 43: 81-89).

Keywords: prognosis score, critical care, cancer, mortality.

\section{Introducción}

El cáncer es la principal causa de muerte en el mundo desarrollado, presentándose 12.7 millones de nuevos casos para el año 2008 (1) y proyectándose 22.2 millones de nuevos casos para el 2030 (2). Sin embargo, los avances en el tratamiento de las neoplasias han mejorado las tasas de supervivencia de esta población, alcanzando remisión y curación en varias patologías, aunque estos adelantos a menudo se logran a través de terapias agresivas (3). llevando a mayor estancia hospitalaria e ingresos a la unidad de cuidado intensivo (UCI) para la atención aguda de la enfermedad, cuidados posoperatorios, o complicaciones del cáncer y su tratamiento (4).

La admisión de pacientes oncológicos a UCI puede estar rodeada de incertidumbre con respecto al impacto de la misma sobre su pronóstico (5). teniendo en cuenta su alta mortalidad que puede llegar incluso al $53 \%$. siendo mayor en pacientes con trasplante de médula ósea, necesidad de ventilación mecánica y choque séptico $(6,7)$ en comparación con la mortalidad de otras patologías graves y crónicas $(8,9)$.

Recientemente, un estudio multicéntrico realizado con la base de datos holandeses National Intensive Care Evaluation (NICE) indicó que la mortalidad a 60 días en pacientes con neoplasias hematológicas fue similar a la de personas con tumores sólidos y también en pacientes con otras comorbilidades graves, tales como insuficiencia cardiaca crónica, cirrosis hepática y enfermedad pulmonar obstructiva crónica (EPOC) (10).

Alrededor del mundo se han venido desarrollando diferentes puntajes pronósticos como Acute Physiology and Chronic Health Evaluation (APACHE IV). Simplified Acute Physiologic Score (SAPS III). Cancer Mortality Model (CMM). The Mortality Prediction Model (MPM II) a los que se les han incluido criterios oncológicos que bien pueden subestimar o sobreestimar la mortalidad (11); por lo cual resulta apropiada la evaluación y comparación de su desempeño en pacientes colombianos de acuerdo con las características propias del contexto.

Existen diferentes puntajes predictivos que han hecho una aproximación a la predicción de mortalidad en este subgrupo de pacientes a través de variables específicas del estado de avance y tratamiento del cáncer como APACHE
IV que es la versión más reciente de este modelo y MPM II; sin embargo, en el país sigue siendo ampliamente utilizado APACHE II que es una versión anterior y menos precisa para subgrupos específicos como cáncer (12). Por lo tanto el objetivo de este estudio es evaluar el desempeño pronóstico de los puntajes Acute Physiology and Chronic Health Evaluation (APACHE IV) y The Mortality Prediction Model (MPM II). para la predicción de mortalidad a siete días en pacientes oncológicos críticamente enfermos admitidos a la unidad de cuidado intensivo (UCI) durante enero de 2014 a enero de 2016.

\section{Material y métodos \\ Tipo y diseño del estudio}

Estudio de tipo observacional analítico de cohorte: se trata de una investigación en la que no se realiza intervención sobre la población en estudio, en el que se exploran relaciones entre variables y se prueba una hipótesis que surge de estudios previos.

\section{Población}

Registros clínicos de pacientes con diagnóstico oncológico ingresados a la unidad de cuidado intensivo de una institución prestadora de servicios de salud especializada en oncología en la ciudad de Bogotá durante enero de 2014 a enero de 2016.

\section{Diseño muestral}

Se tomaron todos los registros de pacientes ingresados a la UCI con diagnóstico de cáncer de los dos últimos años.

Para el cálculo del tamaño de muestra se utilizó fórmula de Freeman: $[\mathrm{n}=10 *(\mathrm{k}+1)]$ lo cual indica que el número de sujetos en un estudio con regresión logística debe ser superior a $10 *(\mathrm{k}+1)$. donde $\mathrm{k}$ expresa el número de covariables.

\section{Criterios de inclusión y exclusión}

Criterios de inclusión

- Registros de pacientes mayores de 18 años de edad.

- Diagnóstico oncológico con conformación histopatológica.

- Para pacientes con reingresos durante el periodo de estudio se tomó el ingreso más reciente y

- Estancia mayor de cuatro horas en la UCI 
Criterios de exclusión

- Readmisión de otra UCI.

- Información incompleta en las variables medidas.

- Gran quemado.

- Diagnóstico de evento coronario.

- Trauma.

- Trasplantados

Variables: se tomaron las variables con las cuales se validaron los modelos pronósticos en sus estudios originales (Figura 1).

\section{Métodos \\ Técnicas de recolección de información}

Fuente de información. La fuente de información es secundaria, los datos de cada paciente fueron obtenidos de forma directa de la historia clínica física y electrónica institucional.

Proceso de obtención de la información. Para la recolección de la información de cada paciente se diseñó un formato con los datos de identificación, las características sociodemográficas y las variables clínicas ya validadas para el cálculo de cada puntaje de predicción de mortalidad.

La recolección de la información de cada paciente fue realizada por el investigador principal, consultando en la historia clínica electrónica institucional, se utilizó el censo de la unidad de cuidado intensivo de enero de 2014 a enero de 2016 para identificar los pacientes que tenían ingreso durante este periodo y que tuvieran diagnóstico oncológico. Para determinar el estado a siete días de la variable desenlace de mortalidad se realizó revisión de la historia de UCI y para aquellos pacientes que habían sido dados de alta antes de completar el tiempo de seguimiento, se indagó en los registros del servicio tratante, unidad de crónicos, consulta externa, laboratorio clínico y ayudas diagnósticas.

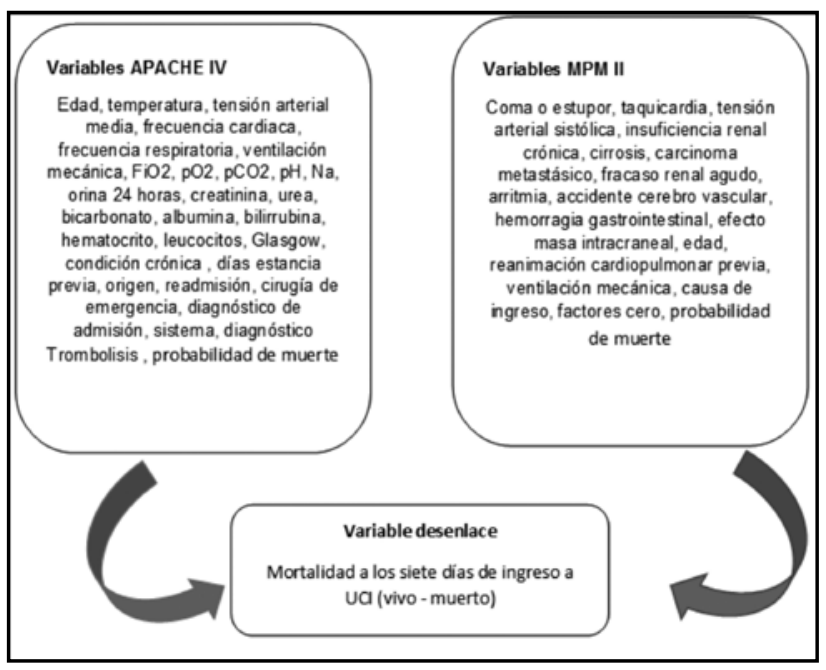

Figura 1. Diagrama de variables.

\section{Análisis estadístico}

En el análisis univariado para las variables cualitativas se reportan proporciones y para las variables cuantitativas se reportan medidas de tendencia central media, desviación estándar y coeficiente de variación y para las variables que no siguen distribución normal se reportaron medianas y cuartiles 1 y 3 .

En el análisis bivariado para las variables cualitativas se construyeron tablas de contingencia y prueba de asociación con el estadístico de $X^{2}$, para las variables cuantitativas se utilizó t de Student para la comparación de promedios y para las variables que no siguen distribución normal se reporta $U$ Mann Whitney para la comparación de medianas.

Para el análisis multivariado se estimó un modelo de regresión logística binaria al cual se ingresaron las variables predictivas ya validadas para cada puntaje pronóstico.

Para determinar la calibración de cada modelo se llevó a cabo la prueba de bondad de ajuste de Hosmer y Lemeshow, que comprueba si el modelo propuesto explica los datos observados, a través de la evaluación de la distancia entre los datos observados en el estudio y lo esperado que es el valor teórico calculado mediante el modelo construido, se comparan observados y esperados en 10 categorías correspondientes aproximadamente a los deciles de riesgo o probabilidad predicha de presentar el evento. Las dos distribuciones se comparan mediante una prueba de $X^{2}$ con 8 grados de libertad (13).

Para evaluar la capacidad discriminativa del modelo se calculó el área bajo la curva ROC con el estadístico C, a través de la construcción de la curva ROC es posible determinar el poder predictivo y discriminación del modelo, se presenta en un diagrama de dos ejes, donde el eje $\mathrm{X}$ es el complementario de la especificidad (1- especificidad) y el eje Y es la sensibilidad. Por lo tanto es una forma de presentar ambas características del modelo logístico en todos los puntos de corte (13).

Para determinar la eficiencia del modelo se estimaron los estadísticos criterio de información Akaike (AIC) y criterio de información Bayesiano (BIC). Cuando se cuenta con diferentes modelos con sus respectivos parámetros o variables es utilizada para su comparación la función de máxima verosimilitud ((likelihood). Esta función permite determinar el modelo que realiza el mejor ajuste de los datos, sin embargo no penaliza su complejidad, por lo cual resulta útil emplear medidas de contraste como el AIC y el BIC. Los criterios hacen uso del Log-likelihood (log Lik). que es el logaritmo de máxima verosimilitud, y sustraen un término proporcional al número de parámetros $(K)$ en el modelo, así: $\log L i k \alpha K$. donde $\alpha$ corresponde a 2 para el AIC y a $\log (N)$ para el BIC (14-16).

\section{Resultados}

Durante el periodo de estudio fueron admitidos 303 pacientes con diagnóstico oncológico a UCI, de los cuales $283(93.4 \%)$ cumplieron los criterios de inclusión y tuvieron información clínica completa (Figura 2). 


\section{Características clínicas de los pacientes y comprobación de asociación entre variables}

Se realizó la descripción de las características clínicas basales de los pacientes que fueron validadas como predictores en los estudios originales para la construcción de los puntajes pronósticos APACHE IV y MPM II (17.18) Encontrando diferencias significativas entre el grupo de pacientes que sobrevivieron y los que fallecieron durante los primeros siete días de estancia en UCI en las siguientes variables: edad, siendo llamativo que el grupo que presentó mortalidad tenía un promedio de edad menor con una diferencia de seis años con respecto a los que vivieron, tanto la frecuencia cardiaca como la respiratoria se encontraron elevadas en el grupo de fallecidos; las variables de los gases arteriales mostraron ser un predictor clave de mortalidad dado que los pacientes que murieron mostraron alteración del estado de oxigenación reflejado en estos parámetros, requerir ventilación mecánica también mostró ser un indicador de mal pronóstico igualmente, el estado de inmunosupresión sugiere que los sujetos que presentan esta condición clínica tienen mayor probabilidad de fallecer, al igual que tener un diagnóstico de admisión quirúrgico de cualquier tipo, como se muestra en la Tabla 1.

Se estimó un modelo de regresión logística binaria para el puntaje APACHE IV en la que se incluyeron todas las variables usadas para la validación del puntaje en su versión original, encontrando que para la población en estudio mostraron significancia estadística las variables edad, frecuencia cardiaca, $\mathrm{FiO}_{2}$, los parámetros de gases arteriales, creatinina, BUN, albúmina, bilirrubina, hematocrito, diagnóstico de admisión médico y desarrollar sepsis, validando que son predictores clínicos de mortalidad en esta cohorte (Tabla 2).

Se estimó un modelo de regresión logística binaria en la que se incluyeron todas las variables usadas para la validación de la escala MPM II en su versión original, encontrando que para la población en estudio mostraron significancia estadística las variables taquicardia, edad, ventilación mecánica y admisión por causa médica, validando que son predictores de mortalidad en concordancia con el modelo para APACHE IV (Tabla 3).

Para determinar el modelo con mejor desempeño se utilizaron los estadísticos de Hosmer y Lemeshow, la comparación de AUC que se muestra en la Figura 3 y la prueba de homogeneidad, además de los coeficientes de información de Akaike (AIC) y coeficiente de información Bayesiano (BIC). como se muestra en la Tabla 4.

La Figura 3 muestra que APACHE IV tiene un mayor poder predictivo dado por mayor área bajo la curva y que se evidencia con curva ROC situada más cerca de la esquina superior izquierda

De acuerdo con los resultados que se muestran en la Tabla 4 el puntaje pronóstico con mayor capacidad predictiva es el APACHE IV dado por una mayor AUC con prueba de homogeneidad de áreas estadísticamente significativa en comparación con MPM II, con respecto a la calibración evaluada con estadístico de Hosmer y Lemeshow no se encontraron diferencias estadísticamente significativas entre los valores observados versus los esperados para ninguno de los modelos.

Para determinar el modelo con mayor eficiencia la Tabla 3 reporta el estadístico BIC para los dos modelos, siendo mayor para APACHE IV, lo que sugiere una menor eficiencia dado que MPM II utiliza un menor número de variables para realizar su predicción; sin embargo, el $\Delta \mathrm{BIC}$ es de 2.9 que muestra evidencia positiva en contra de este modelo pero no fuerte, es decir, que la diferencia del estadístico entre los dos modelos no es significativa, teniendo como referencia que diferencias mayores a seis entre los estadísticos BIC de dos modelos en comparación indican que el modelo con mayor valor muestra evidencia fuerte que indica ser menos eficiente.

También se reporta el estadístico AIC siendo menor para APACHE IV, lo que indica que dicho modelo realiza el mejor ajuste de los datos.

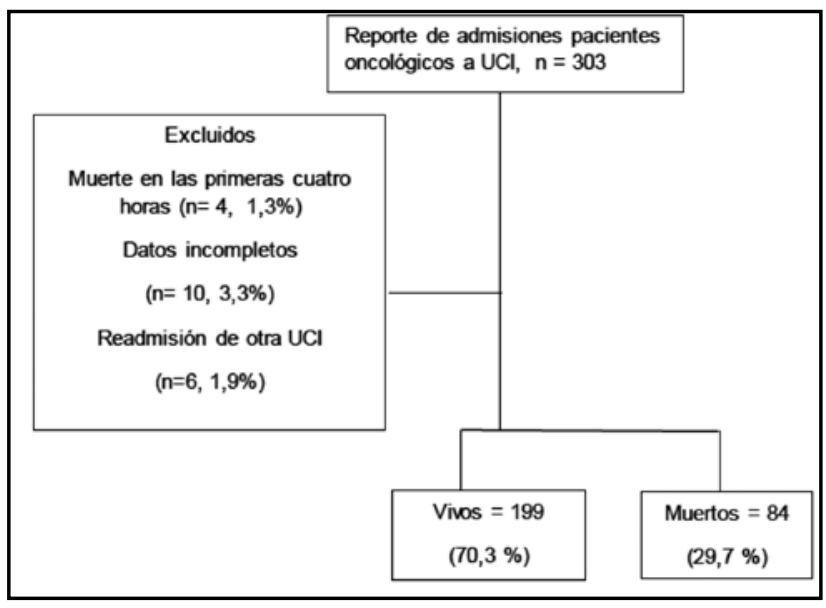

Figura 2. Población del estudio.

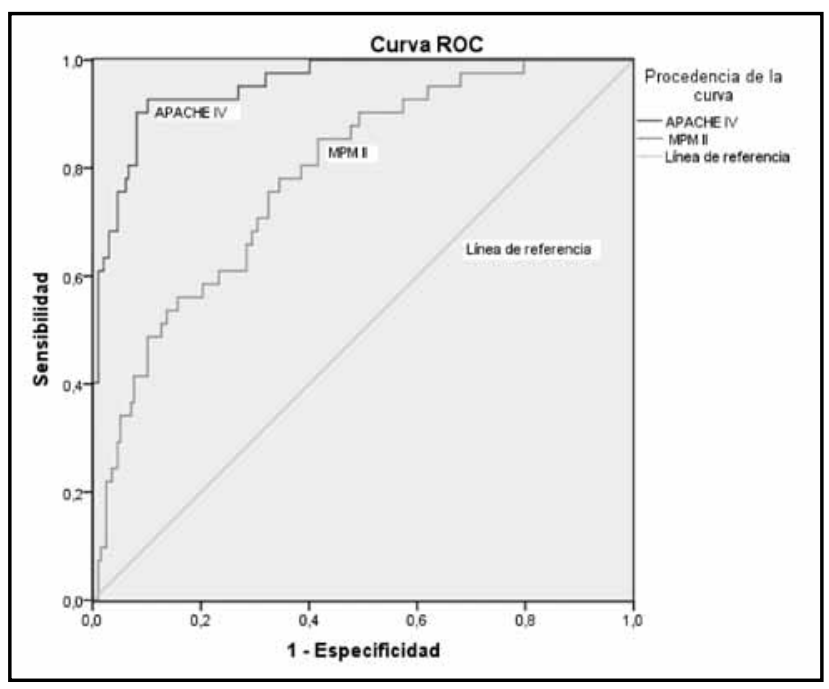

Figura 3. Comparativo curvas ROC APACHE IV Y MPM II. 
TRABAJOS ORIGINALES • Predictores de mortalidad oncológica en UCI

Tabla 1. Características clínicas de los pacientes.

\begin{tabular}{|c|c|c|c|c|c|}
\hline \multirow{2}{*}{ Variables clínicas } & \multicolumn{2}{|c|}{ Muertos $(n=84)$} & \multicolumn{2}{|c|}{ Vivos $(n=199)$} & \multirow{2}{*}{ Valor $\mathbf{p}$} \\
\hline & Media & Desviación estándar & Media & Desviación estándar & \\
\hline Edad & 5469 & 1888 & 60.45 & 15.33 & 0.08 \\
\hline Temperatura & 36.83 & 0.58 & 36.78 & 0.62 & 0.49 \\
\hline TAS & 118.12 & 28.05 & 121.89 & 27.71 & 0.3 \\
\hline TAM & 82.82 & 20.98 & 85.99 & 19.6 & 0.22 \\
\hline $\mathrm{FC}$ & 110.81 & 20.96 & 95.62 & 24.73 & 0.000 \\
\hline FR & 22.18 & 10.2 & 19.72 & 5.24 & 0.005 \\
\hline $\mathrm{FiO}_{2}$ & 44.9 & 19.28 & 39.56 & 13.23 & 0.8 \\
\hline $\mathrm{PaO}_{2}$ & 84.88 & 48.94 & 77.26 & 24.97 & 0.000 \\
\hline $\mathrm{PCO}_{2}$ & 34.12 & 14.78 & 32.35 & 9.15 & 0.002 \\
\hline $\mathrm{pH}$ & 7.28 & 0.18 & 7.4 & 0.1 & 0.000 \\
\hline $\mathrm{HCO}_{3}$ & 17.71 & 7.25 & 21.51 & 9.83 & 0.002 \\
\hline $\mathrm{Na}$ & 140.52 & 7.66 & 141 & 6.31 & 0.58 \\
\hline Albúmina & 2.4 & 0.7 & 2.65 & 0.66 & 0.5 \\
\hline Hematocrito & 31.25 & 6.46 & 30.97 & 7.52 & 0.76 \\
\hline \multirow[t]{2}{*}{ Glucosa } & 92.8 & 18.02 & 93.91 & 19.3 & 0.029 \\
\hline & Mediana & Rango IQ & Mediana & Rango IQ & \\
\hline Orina 24 horas * & 960 & 1716 & 1440 & 1819 & 0.87 \\
\hline Creatinina* & 0.94 & 2 & 0.8 & 1 & 0.63 \\
\hline BUN* $^{*}$ & 23.7 & 36 & 19.8 & 19 & 0.12 \\
\hline Bilirrubina Total* & 1.2 & 1 & 0.7 & 1 & 0.78 \\
\hline Leucocitos* & 15440 & 15390 & 13400 & 11310 & 0.67 \\
\hline Días estancia previa* & 5 & 11 & 4 & 7 & 0.22 \\
\hline \multirow[t]{2}{*}{ Glasgow } & 15 & 12 & 9 & 12 & 0.02 \\
\hline & $\mathbf{N}$ & $\%$ & $\mathbf{n}$ & $\%$ & \\
\hline Sexo & & & & & 0.58 \\
\hline Hombre & 38 & 45.2 & 83 & 41.7 & \\
\hline Mujer & 46 & 54.8 & 116 & 58.3 & \\
\hline Ventilación mecánica & 72 & 85.7 & 120 & 60.3 & 0.000 \\
\hline Falla renal crónica/HD & 4 & 4.8 & 14 & 7 & 0.47 \\
\hline Cirrosis & & & 4 & 2 & 0.19 \\
\hline Falla hepática & 2 & 2.4 & 2 & 1 & 0.37 \\
\hline Carcinoma metastásico & 42 & 50 & 84 & 42.3 & 0.22 \\
\hline Linfoma & 4 & 4.8 & 12 & 6 & 0.67 \\
\hline Leucemia/mieloma & 8 & 9.5 & 22 & 11.1 & 0.7 \\
\hline Inmunosupresión & 8 & 9.5 & & & 0.000 \\
\hline \multicolumn{6}{|l|}{ Origen } \\
\hline Piso & 42 & 50 & 73 & 36.7 & \\
\hline Otro hospital & 30 & 35.7 & 75 & 37.7 & \\
\hline Recuperación cirugía & 12 & 14.3 & 47 & 23.6 & \\
\hline Otro & & & 4 & 2 & \\
\hline Diagnóstico de admisión & & & & & 0.000 \\
\hline Médico & 68 & 81 & 117 & 58.8 & \\
\hline Quirúrgico & 16 & 19 & 82 & 41.2 & \\
\hline \multicolumn{6}{|l|}{ Sistema } \\
\hline Cardiovascular & 6 & 7.1 & 11 & 5.5 & 0.000 \\
\hline Respiratorio & 28 & 33.3 & 41 & 20.6 & \\
\hline Digestivo & 16 & 19 & 55 & 27.6 & \\
\hline Neurológico & 4 & 4.8 & 13 & 6.5 & \\
\hline Metabólico & & & 9 & 4.5 & \\
\hline Hematológico & 10 & 11.9 & 30 & 15.1 & \\
\hline Genitourinario & 8 & 9.5 & 17 & 8.5 & \\
\hline Sepsis & 12 & 14.3 & 19 & 9.5 & \\
\hline Otro & & & 2 & 1 & \\
\hline
\end{tabular}


Tabla 2. Modelo logístico Apache IV.

\begin{tabular}{|c|c|c|c|c|c|}
\hline \multirow{2}{*}{ Variables } & \multirow{2}{*}{ B } & \multirow{2}{*}{$p$} & \multirow{2}{*}{ OR } & \multicolumn{2}{|c|}{ I.C. $95 \%$ para OR } \\
\hline & & & & Inferior & Superior \\
\hline Edad & -.064 & .001 & .938 & .901 & .976 \\
\hline Temperatura & .039 & .934 & 1.04 & .411 & 2.63 \\
\hline TAM & -.002 & .913 & .998 & .968 & 1.02 \\
\hline FC & .033 & .046 & 1.03 & 1.001 & 1.06 \\
\hline FR & -.072 & .193 & .931 & .836 & 1.03 \\
\hline Ventilación mecánica & 2.10 & .038 & 8.17 & 1.121 & 59.64 \\
\hline $\mathrm{FiO} 2$ & .052 & .003 & 1.05 & 1.017 & 1.09 \\
\hline p02 & .025 & .020 & 1.02 & 1.004 & 1.04 \\
\hline $\mathrm{Pco} 2$ & .212 & .008 & 1.23 & 1.057 & 1.44 \\
\hline $\mathrm{PH}$ arterial & 9.84 & .079 & 188 & .318 & 1110 \\
\hline $\mathrm{HCO} 3$ & -.444 & .009 & .641 & .459 & .897 \\
\hline $\mathrm{Na}$ & -.007 & .864 & .993 & .919 & 1.07 \\
\hline Orina Total 24 & .000 & .468 & 1.00 & .999 & 1.00 \\
\hline Creatinina & -.725 & .012 & .484 & .275 & .854 \\
\hline BUN & .051 & .004 & 1.053 & 1.017 & 1.09 \\
\hline Albumina & -1.366 & .011 & .255 & .089 & .729 \\
\hline Bilirrubina total & .354 & .006 & 1.425 & 1.106 & 1.83 \\
\hline Hematocrito & .195 & .001 & 1.216 & 1.079 & 1.37 \\
\hline Glóbulos blancos & .000 & .522 & 1.000 & 1.000 & 1.00 \\
\hline GLASGOW & .115 & .108 & 1.122 & .975 & 1.29 \\
\hline Falla renal crónica / HD & -.447 & .706 & .640 & .063 & 6.52 \\
\hline Cirrosis & -20.00 & .999 & .000 & .000 & . \\
\hline Falla Hepática & 20.991 & .998 & 13064 & .000 & . \\
\hline Carcinoma Metastásico & .674 & .281 & 1.961 & .576 & 6.67 \\
\hline Linfoma & .722 & 672 & 2.058 & .073 & 58.28 \\
\hline Leucemia mieloma & 2.890 & .140 & 17.99 & .387 & 835.5 \\
\hline Inmunosupresión & 23.180 & .998 & 11662 & .000 & . \\
\hline Origen (Otro Hospital) & & .601 & & & \\
\hline Piso & .032 & .963 & 1.033 & .262 & 4.06 \\
\hline Recuperación cirugía & 2.785 & .203 & 16.20 & .223 & 1179.3 \\
\hline Otro & -19.381 & .999 & .000 & .000 & . \\
\hline Días estancia previa & .007 & .806 & 1.007 & .950 & 1.06 \\
\hline Cirugía Emergencia & -17.834 & .999 & .000 & .000 & . \\
\hline Diagnostico Admisión (quirúrgico) & 4.968 & .031 & 14.6 & 1.573 & 131.5 \\
\hline Sistema (Trauma) & & .198 & & & \\
\hline Cardiovascular & -.872 & .489 & .418 & .035 & 4.92 \\
\hline Digestivo & -.311 & .728 & .733 & .127 & 4.22 \\
\hline Neurológico & .807 & .506 & 2.24 & .207 & 24.2 \\
\hline Metabólico & -44.099 & .997 & .000 & .000 & . \\
\hline Hematológico & -3.549 & .061 & .029 & .001 & 1.17 \\
\hline Genitourinario & 1.915 & .115 & 6.78 & .628 & 73.3 \\
\hline Sepsis & -3.082 & .010 & .046 & .004 & .485 \\
\hline Otro & -23.252 & .999 & .000 & .000 & . \\
\hline
\end{tabular}


TRABAJOS ORIGINALES • Predictores de mortalidad oncológica en UCI

Tabla 3. Modelo logístico MPM II.

\begin{tabular}{|c|c|c|c|c|c|}
\hline \multirow{2}{*}{ Variables } & \multirow{2}{*}{ B } & \multirow{2}{*}{ Sig. } & \multirow{2}{*}{ OR } & \multicolumn{2}{|c|}{ I.C. $95 \%$ para OR } \\
\hline & & & & Inferior & Superior \\
\hline Coma & .198 & .647 & 1.21 & .521 & 2.85 \\
\hline Taquicardia $\geq 150 \mathrm{lpm}$ & .016 & .015 & 1.01 & 1.00 & 1.02 \\
\hline TAS $90 \mathrm{mmHg}$ & -.003 & .595 & .997 & .987 & 1.00 \\
\hline Falla renal crónica / HD & -.569 & .377 & .566 & .160 & 2.00 \\
\hline Cirrosis & -19.55 & .999 & .000 & .000 & . \\
\hline Carcinoma metastásico & .506 & .115 & 1.65 & .884 & 3.11 \\
\hline Arritmia & -.373 & .727 & .688 & .085 & 5.58 \\
\hline Accidente cerebrovascular & -.466 & 1.00 & .627 & .000 & . \\
\hline Hemorragia gastrointestinal & -.154 & .892 & .857 & .093 & 7.87 \\
\hline Efecto Masa intracraneal & -19.93 & .999 & .000 & .000 & . \\
\hline Edad & -.020 & .027 & .980 & .963 & .998 \\
\hline $\mathrm{RCP}$ previa al ingreso & -.119 & .846 & .888 & .268 & 2.93 \\
\hline Ventilación Mecánica & 1.29 & .001 & 3.64 & 1.693 & 7.86 \\
\hline Causa médica & .943 & .010 & 2.56 & 1.252 & 5.27 \\
\hline Cirugía no programada & -20.15 & .999 & .000 & .000 & . \\
\hline
\end{tabular}

Tabla 4. Comparación de los modelos.

\begin{tabular}{|l|c|c|}
\hline \multicolumn{1}{|c|}{ Estadístico } & Apache IV & MPM II \\
\hline H-L & $P=0.398$ & $p=0.991$ \\
\hline AIC & 235.4 & 317.1 \\
\hline BIC & 13.3 & 10.4 \\
\hline$\Delta$ BIC & $0.955 \quad 2.9$ \\
\hline AUC & (IC=0.93-0.97) & $\begin{array}{c}0.787 \\
(I C=0.73-0.84)\end{array}$ \\
\hline Homogeneidad de áreas & \multicolumn{2}{|c|}{ P $\leq 0.0000$} \\
\hline
\end{tabular}

\section{Discusión}

Este estudio observacional analítico de cohorte en pacientes oncológicos críticos manejados en UCI a quienes se les estimó la probabilidad de mortalidad con los puntajes pronósticos APACHE IV y MPM II. Mostró que APACHE IV tiene un buen desempeño en la predicción de mortalidad de pacientes oncológicos críticamente enfermos. APACHE IV se reitera y se constituye en una herramienta útil para el clínico en su labor diaria, al permitirle distinguir los pacientes con alta probabilidad de mortalidad.

Se han desarrollado modelos específicos para predicción de mortalidad en pacientes con cáncer; sin embargo, es un desafío la implementación en UCI dado que tienden a subestimar la probabilidad de muerte que podría estar dado por la propia heterogeneidad de la enfermedad maligna. Además de generar controversias como el hecho de si un tumor sólido y una neoplasia hematológica son demasiado diferentes para un único sistema de puntuación y si el cáncer no es un factor de pobre pronóstico per se y por tanto no es una razón de exclusión para la admisión de estos pacientes a UCI (19).

Con respecto a los resultados de nuestro estudio, los puntajes pronósticos mostraron una adecuada calibración
(Test de Hosmer y Lemeshow no significativo) de los modelos, resultados que son discordantes con otros estudios donde han reportado pobre calibración como el de George (20), en el cual se derivó un modelo específico para cáncer y el de Soares en el cual se compararon seis puntaje entre los que se incluyen MPM II ( $\mathrm{p}=0.001)$ y APACHE III ( $\mathrm{p}$ $=0.001$ ) con CMM que es un modelo derivado específicamente para pacientes oncológicos críticamente enfermos (11). Sin embargo, en el estudio de derivación de APACHE IV se reportó adecuada calibración $(\mathrm{p}=0.08)(17)$. no se encontraron estudios adicionales donde se compara esta versión reciente de APACHE con otros puntajes.

En el presente estudio la evaluación del poder predictivo a través del estadístico C y AUC, sugiere que los dos puntajes pronósticos tienen adecuada capacidad de predicción de acuerdo con su capacidad de discriminación, aunque, APACHE IV mostró mayor AUC (0.95) que MPM II (0.78) y que es corroborada al realizar prueba de homogeneidad de áreas que muestra diferencias estadísticamente significativas, estos hallazgos son concordantes con el estudio de Zimmerman (17) en el cual se validaron las variables predictoras para APACHE IV el cual reporta adecuada AUC (0.88). Para MPM II Soares en una de sus publicaciones reporta un AUC (0.73) (11) cercana a nuestros resultados; sin embargo, en otro estudio de Groeger (20), MPM II no mostró buena capacidad predictiva con AUC (0.63) con menor desempeño con relación a nuestra evaluación de los puntajes.

La eficiencia de los modelo fue evaluada con los estadísticos AIC y BIC encontrado un BIC superior para APACHE IV lo que inicialmente sugiere un modelo menos eficiente con respecto a MPM II, ya que requiere de múltiples parámetros para realizar su predicción y al evaluar el $\Delta$ BIC aunque se presenta una diferencia positiva con evidencia contra APACHE IV dicha diferencia no llega a ser una evidencia fuerte contra el modelo (21). 
Con respecto al estadístico AIC fue inferior para APACHE IV en comparación con MPM II, lo que sugiere que es un modelo con buen ajuste a los datos observados, es decir, explica gran parte de la variabilidad de los datos, aunque complejo por el gran número de parámetros utilizados.

Estas diferencias encontradas entre los resultados del BIC y AIC de ambos modelos y cuya interpretación es similar, estaría explicada por el término de penalización por el número de variables del modelo que es mayor en el BIC y que tiene en cuenta no sólo la cantidad de parámetros sino el tamaño de la muestra. No se encontraron estudios relacionados con puntajes pronósticos comparativos en el uso de estos estadísticos.

Esta investigación encontró como ventaja el hecho de no requerir para el uso de MPM II el ingreso de un diagnóstico específico, por lo cual no es necesario seleccionar entre varios diagnósticos principales o importantes en un paciente complejo, previniendo errores en la clasificación y disminuyendo la afectación sobre la calibración. Por otra parte proporciona una evaluación aguda a través de la variable edad y quince variables binarias en el momento del ingreso o en la primera hora de admisión a la UCI dado que no requiere de reportes de ayudas diagnósticas, mientras que APACHE IV se basa en gran parte en los peores valores de los parámetros fisiológicos obtenidos en las primeras 24 horas del ingreso.

Por lo tanto MPM II está basado en la condición y estado clínico del sujeto previo al inicio de la atención y cuidado en la unidad crítica, lo que resulta de gran utilidad, ya que permite estimar la calidad del cuidado mediante una evaluación del paciente ajustada al riesgo estimado de mortalidad y los resultados de la atención.

Se evidenciaron limitaciones de MPM II para pacientes que tienen cambios rápidos en su estado vital al ser admitidos y recibir atención, en razón a que no tiene en cuenta la evolución de las primeras horas y el impacto de la atención sobre el estado clínico. Adicionalmente no tiene en cuenta subgrupos de riesgo como cirugía cardiaca, infarto agudo de miocardio y reingreso a la UCI, lo que reduce su utilidad en algunas UCI. También se encontró que la discriminación de MPM II es inferior en comparación con APACHE IV, por lo que este modelo requiere de nuevas revisiones $\mathrm{y}$ adaptaciones.

Por otra parte. APACHE IV se desarrolló debido a que la exactitud de APACHE II ha sufrido cambios significativos en la década previa, en esta investigación APACHE IV mostró una alta discriminación y buena calibración incorporando un gran número de variables, este aumento en la complejidad de APACHE IV denota un cambio significativo en la simplificación de las versiones anteriores, sin embargo, la puntuación APACHE II continúa siendo una medida resumen útil de gravedad de la enfermedad.

El aumento en la complejidad de los puntajes pronósticos de mortalidad por un mayor número de variables predictoras podría llegar a ser una desventaja en la práctica al requerir experticia y tiempo de los profesionales para su aplicación rutinaria en las UCI, por lo que se requiere el desarrollo y utilización de herramientas tecnológicas que permitan la extracción de la información de forma práctica y ágil para los profesionales.

Los modelos pronósticos para pacientes de UCI en general tienden a subestimar el riesgo de muerte en pacientes con cáncer críticamente enfermos. Esto es importante cuando se interpretan tasas de mortalidad como medida de comparación entre unidades, ya que unidades con mayor ocupación por pacientes oncológicos tienden a presentar una mayor tasa de mortalidad. Sin embargo, estos modelos tienen la capacidad de identificar subgrupos de pacientes con alto riesgo.Por lo tanto puede tener un papel importante para la asignación de recursos y proveer información sobre el pronóstico para personal de atención, pacientes y familiares (22).

En conclusión este estudio observacional analítico de cohorte en pacientes oncológicos críticos manejados en UCI a quienes se les estimó la probabilidad de mortalidad con los puntajes pronósticos APACHE IV y MPM II, mostró que APACHE IV tiene un buen desempeño en la predicción de mortalidad de pacientes oncológicos críticamente enfermos. APACHE IV se reitera y se constituye en una herramienta útil para el clínico en su labor diaria, al permitirle distinguir los pacientes con alta probabilidad de mortalidad. En este orden de ideas, es importante destacar la importancia de continuar desarrollando herramientas tecnológicas que permitan la extracción de la información de forma práctica y ágil para los profesionales en pacientes oncológicos críticos a tratar en UCI .

\section{Referencias}

1. Ferlay J. Shin H. Bray F. Forman D. Mathers C. Parkin D. Estimates of worldwide burden of cancer in 2008: GLOBOCAN 2008. Int J Cancer. 2010 Diciembre; 127 (12).

2. Freddie B. Ahmedin J. Nathan G. Jacques F. David F. Global cancer transitions according to the Human Development Index (2008-2030): a population-based study. Lancet Oncol. 2012 Agosto; 13 (8).

3. David S. James S. Douglas B. Wolff P. Anne J. Intensive care, survival, and expense of treating critically ill cancer patients. JAMA. 1993 Febrero; 6 (269).

4. James S. Intensive care and oncology. Supp Care Cancer. 1995 Marzo; 3 (2).

5. Azoulay É. Pochard F. Chevret S. Vinsonneau C. Compliance with triage to intensive care recommendations. Crit Care Med. 2001 Noviembre; 29 (11).

6. Benoit D. Vandewoude K. Decruyenaere J. Hoste E. Colardyn F. Outcome and early prognostic indicators in patients with a hematologic malignancy admitted to the intensive care unit for a life-threatening complication. Crit Care Med. 2003 Enero; 31 (1).

7. Staudinger T. Stoiser B. Müllner M. Locker G. Laczika K. Knapp S. Outcome and prognostic factors in critically ill cancer patients admitted to the intensive care unit. Crit Care Med. 2000 Mayo; 28 (5).

8. Wachter R. Luce J. Hearst N. Lo B. Decisions about resuscitation: inequities among patients with different diseases but similar prognoses. Ann Intern Med. 1989 Septiembre; 111 (6).

9. Tanvetyanon T. Leighton J. Life-sustaining treatments in patients who died of chronic congestive heart failure compared with metastatic cancer. Crit Care Med. 2003 Enero; 31 (1).

10. Vliet Mv. Verburg I. Boogaard M. Keizer N. Trends in admission prevalence, illness severity and survival of haematological patients treated in Dutch intensive care units. Inten Care Med. 2014 Septiembre; 40 (9).

11. Soares M. Fontes F. Dantas J. Performance of six severity-of-illness scores in cancer patients requiring admission to the intensive care unit: a prospective observational study. Crit care. 2004 Mayo; 8 (4). 
12.T. Berghmans MPJPS. Is a specific oncological scoring system better at predicting the prognosis of cancer patients admitted for an acute medical complication in an intensive care unit than general gravity scores? Supp Care Cancer. 2004 Enero; 12.

13. Martinez M. Sánchez A. Aspectos avanzados de regresión logísitica. In Martinez M. editor. Bioestadistica amigable. Madrid: Diaz de Santos; 2009. p. 777- 818.

14.Posada S. Rosero R. Comparación de modelos matemáticos: una aplicación en la evaluación de alimentos para animales. Rev. Colomb. Cienc. Pecu. 2007 Mayo; 20.

15. Gómez S. Torres V. García Y. Navarro J. Procedimientos estadísticos más utilizados en el análisis de medidas repetidas en el tiempo en el sector agropecuario. Rev Cubana Cienc Agríc. 2012 Diciembre; 16 (1).

16. L G. Tesis de maestria. Matrices de covarianza estructuradas en modelos con medidas repetidas. Recinto universitario de Mayagüez. Universidad de Puerto Rico. 2005.
17.Zimmerman JE. Kramer AA. Douglas S. McNair. Acute Physiology and Chronic Health Evaluation (APACHE) IV: Hospital mortality assessment for today's critically ill patients. Crit Care Med. 2006 Mayo; 34 (5).

18. Lemeshow S. Le JR. Modeling the Severity of Illness of ICU Patients. JAMA. 1994 Octubre; 272 (13).

19. Farquhar-Smith WP. Wigmore T. Outcomes for patients in critical care. Curre anaesth critic care. $2008 ; 19$.

20.JS G. J G. DM N. Probability of mortality of critically ill cancer patients at 72 $\mathrm{h}$ of intensive care unit (ICU) management. Supp Care cancer. 2003 Noviembre; 11 (310).

21. Kass R. Raftery A. Bayes Factors. J Amer Statist Associ. 1995; 90 (430).

22. Wigmore TJ. Farquhar-Smith P. Lawson A. Intensive care for the cancer patient - Unique clinical and ethical challenges and outcome prediction in the critically ill cancer patient. Best Practice \& Research Clin Anaesthe. 2013 Diciembre; 27 (4). 